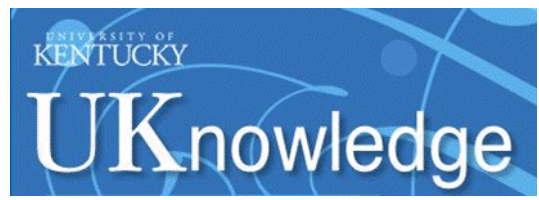

University of Kentucky

UKnowledge

3-28-2018

\title{
The Role of Nutrition in Influencing Mechanisms Involved in Environmentally Mediated Diseases
}

\author{
Bernhard Hennig \\ University of Kentucky, bhennig@uky.edu \\ Michael C. Petriello \\ University of Kentucky, michaelcpetriello@gmail.com \\ Mary V. Gamble \\ Columbia University \\ Young-Joon Surh \\ Seoul National University, South Korea \\ Laura A. Kresty \\ University of Michigan - Ann Arbor \\ Sellow this and dadditionalworks at: https://uknowledge.uky.edu/superfund_facpub \\ Part of the Diseases Commons, Environmental Health and Protection Commons, and the Nutrition \\ Commons \\ Right click to open a feedback form in a new tab to let us know how this document benefits you.
}

\section{Repository Citation}

Hennig, Bernhard; Petriello, Michael C.; Gamble, Mary V.; Surh, Young-Joon; Kresty, Laura A.; Frank, Norbert; Rangkadilok, Nuchanart; Ruchirawat, Mathuros; and Suk, William A., "The Role of Nutrition in Influencing Mechanisms Involved in Environmentally Mediated Diseases" (2018). Superfund Research Center Faculty Publications. 6.

https://uknowledge.uky.edu/superfund_facpub/6

This Review is brought to you for free and open access by the Superfund Research Center at UKnowledge. It has been accepted for inclusion in Superfund Research Center Faculty Publications by an authorized administrator of UKnowledge. For more information, please contact UKnowledge@lsv.uky.edu. 


\section{The Role of Nutrition in Influencing Mechanisms Involved in Environmentally Mediated Diseases}

Digital Object Identifier (DOI)

https://doi.org/10.1515/reveh-2017-0038

Notes/Citation Information

Published in Reviews on Environmental Health, v. 33, issue 1, p. 87-97.

(C)2018 Walter de Gruyter GmbH, Berlin/Boston.

The copyright holder has granted the permission for posting the article here.

Authors

Bernhard Hennig, Michael C. Petriello, Mary V. Gamble, Young-Joon Surh, Laura A. Kresty, Norbert Frank, Nuchanart Rangkadilok, Mathuros Ruchirawat, and William A. Suk 


\title{
Mini Review
}

\author{
Bernhard Hennig*, Michael C. Petriello, Mary V. Gamble, Young-Joon Surh, Laura A. Kresty, \\ Norbert Frank, Nuchanart Rangkadilok, Mathuros Ruchirawat and William A. Suk
}

\section{The role of nutrition in influencing mechanisms involved in environmentally mediated diseases}

https://doi.org/10.1515/reveh-2017-0038

Received October 11, 2017; accepted December 13, 2017; previously published online January 30, 2018

\begin{abstract}
Human exposure to environmental contaminants such as persistent chlorinated organics, heavy metals, pesticides, phthalates, flame retardants, electronic waste and airborne pollutants around the world, and especially in Southeast Asian regions, are significant and require urgent attention. Given this widespread contamination and abundance of such toxins as persistent organic pollutants (POPs) in the ecosystem, it is unlikely that remediation alone will be sufficient to address the health impacts associated with this exposure. Furthermore, we must assume that the impact on health of some of these contaminants results in populations with extraordinary vulnerabilities to disease risks. Further exacerbating risk; infectious diseases, poverty and malnutrition are common in the Southeast Asian regions of the world. Thus, exploring preventive measures of environmental exposure and disease risk through new paradigms of environmental toxicology, optimal and/or healthful nutrition and health is essential. For example, folic acid supplementation can lower blood arsenic levels, and plant-derived bioactive nutrients can lower cardiovascular and cancer
\end{abstract}

*Corresponding author: Bernhard Hennig, University of Kentucky Superfund Research Center, University of Kentucky, Lexington, KY 40536, USA, E-mail: bhennig@uky.edu

Michael C. Petriello: University of Kentucky Superfund Research Center, University of Kentucky, Lexington, KY 40536, USA

Mary V. Gamble: Department of Environmental Health Sciences, Mailman School of Public Health, Columbia University, NY, USA

Young-Joon Surh: Tumor Microenvironment Global Core Research Center, College of Pharmacy, Seoul National University,

Seoul 151-742 08826, South Korea

Laura A. Kresty: Section of Thoracic Surgery, Department of Surgery, University of Michigan, Ann Arbor, MI, 48109, MI, USA

Norbert Frank: German Cancer Research Center, 69120 Heidelberg, Germany

Nuchanart Rangkadilok and Mathuros Ruchirawat: Chulabhorn Research Institute, Bangkok 10210, Thailand

William A. Suk: National Institute of Environmental Health Sciences, National Institutes of Health, Research Triangle Park, NC, USA risks linked to pollutant exposure. Data also indicate that diets enriched with bioactive food components such as polyphenols and omega-3 polyunsaturated fatty acids can prevent or decrease toxicant-induced inflammation. Thus, consuming healthy diets that exhibit high levels of antioxidant and anti-inflammatory properties, is a meaningful way to reduce the vulnerability to non-communicable diseases linked to environmental toxic insults. This nutritional paradigm in environmental toxicology requires further study in order to improve our understanding of the relationship between nutrition or other lifestyle modifications and toxicant-induced diseases. Understanding mechanistic relationships between nutritional modulation of environmental toxicants and susceptibility to disease development are important for both cumulative risk assessment and the design and implementation of future public health programs and behavioral interventions.

Keywords: bioactive foods; environmental pollutants; inflammatory diseases.

\section{Introduction}

The pathologies of non-communicable, chronic diseases such as atherosclerosis, diabetes, obesity and nonalcoholic liver disease are complex and may be influenced by exposure to environmental pollutants throughout the lifespan. Their etiologies are complex and depend on a dynamic interaction between genetics, nutrition, toxicant exposures and the environments where we live. Thus, it is vital that public health professionals working with at-risk populations not only help monitor the ongoing health of exposed communities but also promote healthy practices and behaviors in order to prevent or reduce the potential for disease pathology.

Such at-risk populations can be found in Southeast Asia, which has endured serious environmental health challenges that have emerged primarily from decades of widespread environmental contamination in the entire region, coupled with severe environmental ignorance. Past contaminations resulted from the use of defoliants, 
such as Agent Orange in the Vietnam War, which contributed to great hardship, such as birth defects (1), cancers (2) and skin diseases (3). Other examples include past forest fires in Southeast Asia, as well as continued slash-andburn techniques adopted by farmers in Indonesia, which have been extensively used for many years as the cheapest and easiest means to clear the lands for traditional agriculture. In addition to persistent organic pollutants (POPs), heavy metals and a continued increase in contaminated groundwater and indoor/outdoor air pollution, emerging hazardous wastes, such as electronic waste, have become a major environmental burden for East and Southeast Asia. In addition to the challenges of dealing with environmental pollution, global warming, etc., there is a high incidence of poverty $(4,5)$, viral infection $(6-8)$ and malnutrition $(9,10)$ in this region of the world.

The 8th Princess Chulabhorn International Science Congress, which was held in Bangkok, Thailand in 2016, provided opportunities to develop collaborations that combine population health discoveries generated in Southeast Asian countries with bench research conducted in other parts of the world to advance our overall understanding of environmental health and prevention paradigms. Discussions at this international congress included intervention and/or prevention paradigms against environmental insults and subsequent disease risks. Inspired by these discussions, this review summarizes nutritional intervention and/or prevention paradigms to successfully combat mechanisms involved in diseases linked to deleterious environmental exposures. A particular focus is on POPs, including dioxin-like chemicals such as polychlorinated biphenyls (PCBs), which, due to their chemical nature and persistence in the environment, can generate both shortand long-term health challenges for affected community members. This mini-review will discuss examples by which diet-derived bioactive food components can reduce the risk of pathologies of non-communicable diseases, followed by evidence that such protective nutrients or bioactive food components can modulate or reduce the disease risks associated with exposure to environmental pollutants.

\section{Preventative measures of diet-derived bioactive food components against non- communicable diseases}

Specific foods or natural components within our daily diets can modulate environmental insults and thus the pathologies of non-communicable diseases. The scientific evidence to support the concept of health promoting foods, also referred to as "functional foods", is growing rapidly. Functional foods and herbal supplements contain different types of valuable therapeutic agents. Therefore, the intensive qualitative and quantitative testing of these active ingredients in these products is necessary for their effective utilization and reduction of side effects. In Thailand and other parts of Southeast Asia, people have used herbal products for the treatment of diseases and health promotion for a long time. The following are examples of functional foods or plant-derived bioactive food components used as a means of prevention/intervention against non-communicable diseases.

\section{Sesame and cancer}

Sesame (Sesamum indicum Linn.) has long been used extensively as a traditional food in Eastern countries. Sesame seed and oil are widely used in cooking and as ingredients of confectionery foods. Sesame seeds contain a group of bioactive compounds such as lignans. Sesamin, sesamol and sesamolin, which are major lignans in sesame seed (11), have many pharmacological properties, including antioxidant and anti-proliferative activities, enhancing the antioxidant activity of vitamin $\mathrm{E}$ in the lipid peroxidation system, lowering cholesterol levels, increasing hepatic fatty acid oxidation enzymes, hypotensive effects and neuroprotective effects against hypoxia or brain damage (12). In addition, sesame possesses anticancer properties, which appear to be due mainly to its anti-proliferative, pro-apoptotic, anti-inflammatory and anti-metastatic activities (13). For example, sesamin, a major constituent of sesame, can downregulate the secretion of cytokines and the expression of cell proliferativeand invasive-related gene products in prostate cancer cells (14). Furthermore, sesame lignans and their metabolites (e.g. enterodiol, enterolactone) have been shown to have estrogenic activities in breast cancer T47D cells (15). In addition, frequent consumption of sesame oil was linked to lower risk of stomach cancer in humans (16).

\section{Sesame and cardiovascular diseases}

Air pollution exposure has been linked to cardiovascular disease, with inflammation involved as a suspected mechanism (17). A previous study showed that treatment with an aqueous extract from sesame seeds significantly reversed the changes in all biochemical parameters (notably, lipid 
peroxidation and aspartate aminotransferase) evaluated in serum and heart homogenates of cadmium-induced cardiotoxicity in rats (18). In that study, exposure to cadmium in drinking water caused pronounced oxidative stress and cardiac tissue damage in rats, and an aqueous extract of sesame seeds possessed antioxidant and cardioprotective effects in a dose-dependent manner without the signs of toxicity. Results from a study of dietary black sesame meal on blood pressure and oxidative stress in patients with prehypertension showed that a 4-week administration of black sesame meal significantly decreased systolic blood pressure and plasma malondialdehyde levels, and increased vitamin E levels (19). It is likely that the antihypertensive effect of this black sesame meal is due to decreased oxidative stress and improving antioxidant status in the body (vitamin E levels). In another study with 38 hyperlipidemic patients in Iran, consuming 40 $\mathrm{g}$ of white sesame seeds daily for 60 days was linked to significant decreases in levels of serum total cholesterol (TC), low-density lipoprotein cholesterol (LDL-C) and TC/HDL-C ratio (20). However, serum triglyceride (TG) and high-density lipoprotein (HDL-C) concentrations remained unchanged during the study. It is possible that these lipid changes are related in part to bile acid metabolism, because associations between body fat content, HDL and triglycerides and some secondary bile acid plasma concentrations were found in healthy adults (21).

\section{Longan fruit}

Fruit and vegetables contain many antioxidant compounds, including carotenoids, thiols, glucosinolates, flavonoids and phenolics. An increase in the consumption of antioxidants from these foods can reduce oxidative stress and prevent chronic diseases, including those linked to pollution exposure and associated inflammation. Longan fruit (Dimocarpus longan Lour.) is a subtropical fruit, known as "Lumyai" in Thailand. It is widely grown in China, Taiwan and Southeast Asia including Thailand and Vietnam and is consumed in both fresh and processed products such as canned longan in syrup or dried fruit, as well as fruit drinks. Longan extracts contain high levels of polyphenolic compounds such as corilagin, gallic acid and ellagic acid (22). Dried seed was found to contain the highest levels of these three compounds while dried pulp contained the lowest. Water extracts of longan seed and pulp contained high levels of gallic acid and ellagic acid and exhibited the highest radical scavenging activities (23). These data suggest that longan seed extract could be another potential source of natural dietary antioxidants.
In addition, extracts from the longan fruit exhibited antiinflammatory properties. Mechanisms underlying these effects were related to nitric oxide (NO) scavenging and inhibition of inducible nitric oxide synthase (iNOS) (24). Therefore, longan extracts may be potential natural ingredients for the treatment of inflammatory-related diseases.

\section{Chemoprevention by xanthohumol}

Exposure to persistent organic chemicals has been linked to cancer (25). One possibility of cancer prevention may involve the use of chemicals or dietary components to block, inhibit or reverse the development of cancer in normal or preneoplastic tissues. Prenylated chalcones and flavonoids may be important in cancer prevention. These naturally occurring compounds, which include xanthohumol, exhibit antioxidant and anti-inflammatory properties, and can modulate the metabolism of carcinogens by inhibition of distinct phase 1 metabolic enzymes and activation of phase 2 detoxifying enzymes (26). In a recent human study it was found that xanthohumol can prevent DNA damage by dietary carcinogens, such as 2-amino-3-methylimidazo[4,5-f]quinolone (27), which can be formed during the cooking of meat and fish (28). Also, xanthohumol can have anti-inflammatory actions in cancer cells by suppressing prostaglandin E2 production and cell migration (29). Furthermore, xanthohumol can prevent carbon tetrachloride-induced acute liver injury due to its antioxidant properties (30).

Xanthohumol is a prenylated flavonoid found in hops, which is used in the beer brewing process contributing to the bitterness and the aroma of beer. In addition, many non-alcoholic drinks and foods are rich in xanthohumol, which has implications in human intervention trials. For example, xanthohumol enriched soft drinks and teas are commercially available, as well as muesli bars, chocolate, yogurt and biscuits. A human study was planned in which volunteers consumed nonalcoholic beverages containing xanthohumol or placebo drinks for 2 weeks (31). Alterations of DNA stability was monitored in lymphocytes and a decrease of oxidatively damaged purines was found (31). In addition, a lower formation of reactive oxygen species was observed as monitored by a reduced excretion of 8-oxo-guanosine in urine. Other biochemical parameters reflecting the redox and hormonal status and lipid and glucose metabolism were not altered after the intervention. These data suggest that xanthohumol is a cancer chemopreventive agent in animal studies, and it also can exhibit DNA protecting properties in humans. 


\section{Chemoprevention of aerodigestive tract cancers by berries}

Epidemiological investigations support the notion that increased consumption of diets rich in fruit, vegetables, antioxidants and fiber reduces the risk for cancers of the aerodigestive tract. The inverse association between plantbased diets and cancer has led to evaluations of food-based constituents in a variety of preclinical models to decipher cancer inhibitory mechanisms and potential targets (3235). Promising results have supported early phase clinical trials in cohorts at increased risk for cancer progression due to existing premalignancy or genetic alterations (36-39). Positive effects of cranberries or cranberry-derived constituents have been reported against 16 different cancers (45 cancer cell lines) utilizing a variety of in vitro methods [reviewed in (35)]. Furthermore, in vivo studies support the inhibitory action of cranberries toward cancers of the esophagus, stomach, colon, bladder, prostate, glioblastoma and lymphoma (35). To date, reported mechanisms of cranberry-linked cancer inhibition include cellular death induction via apoptosis, necrosis and autophagy; inhibition of cellular proliferation and modulation of cell cycle kinetics; alterations in reactive oxygen species; as well as modification of cytokine and signal transduction pathways (32-35, 40). Additional proposed mechanisms are summarized in Figure 1 (32, 34, 35). Mechanisms associated with chemoprevention of aerodigestive tract cancers by berries may have therapeutic implications in aerodigestive tract cancer research linked to petroleum exposure. For example, exposure to high levels of sulfur dioxide, nitrogen dioxide, ozone, carbon monoxide, ammonia and particulate matter has contributed to a marked increase in aerodigestive tract diseases especially carcinomas (41). Recent unpublished data support that cranberry proanthocyanidins can inhibit reflux-induced esophageal adenocarcinoma in part via modulation of bile salt and arachidonic acid cascade metabolites and altered microbiome profiles. These findings are in alignment with a limited but growing body of research reporting cranberry modulation of gut microbiota as well as cardiometabolic risk factors $(42,43)$. This body of resesarch is intriguing given that gut bacteria play a large role in modifying not only nutrients but also toxicants, including those found in pollution. Some examples from that body of work: Anhe et al. (43) recently showed that a polyphenol-rich cranberry extract protects from diet-induced obesity, insulin resistance and intestinal

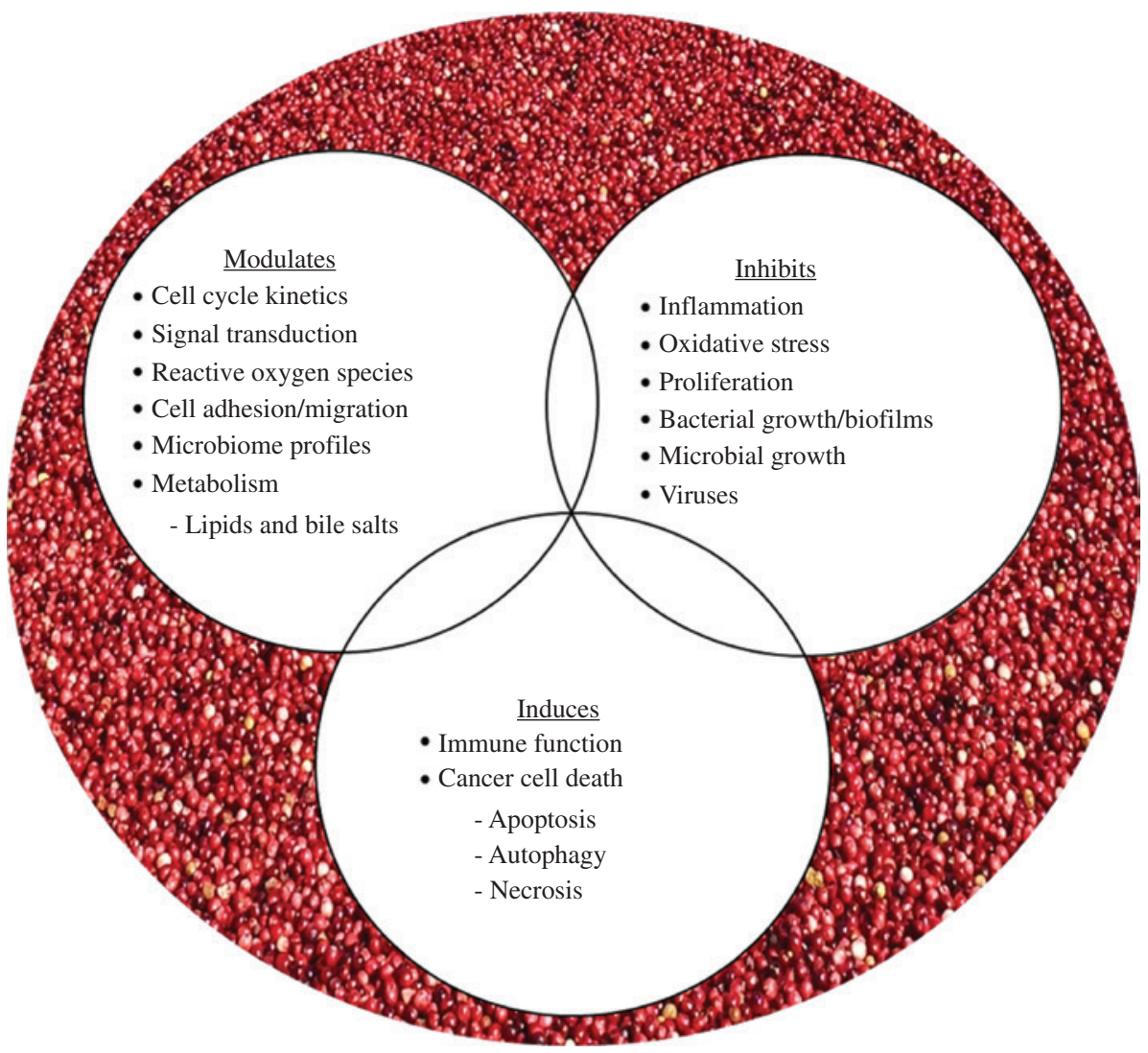

Figure 1: Cancer-inhibitory mechanisms associated with cranberries. 
inflammation in association with increased abundance of the mucin-degrading bacterium Akkermansia in the gut microbiota of mice. Additionally, cranberries and their bioactive constituents modulate bacterial adhesion, coaggregation and biofilm formation which may in turn reduce gastrointestinal and urinary tract infections and serve to reduce systemic inflammation (42). Cranberries have also shown efficacy against Helicobacter pylori (H. pylori), a Gram-negative bacterium linked to peptic ulcer disease, gastric carcinoma and lymphoid tissue lymphoma $(44,45)$.

Bacterial virulence is sensitive to environmental cues including $\mathrm{pH}$ and iron, a limiting nutrient for bacteria during infection. Interestingly, many plant-derived flavonoids, including cranberry proanthocyanidins, contain "iron-binding motifs" which result in strong iron binding properties and cellular iron depletion $(46,47)$. Considering the high metabolic demands of tumors, natural products that target iron metabolism may prove promising for cancer inhibition or as a complement to standard chemotherapy regimens. The antioxidant properties of plant polyphenolics has been widely reported; yet, many of these constituents are redox reactive, forming iron complexes that result in cytotoxic cancer cell death due to the generation of reactive oxygen species $(48,49)$. Cranberry proanthocyanidins have been reported to increase reactive oxygen species (ROS) levels in ovarian, neuroblastoma and esophageal adenocarcinoma cells leading to cancer cell death (35). Whether the chelation capacity of plantderived bioactives extends beyond iron to other heavy metals, such as mercury, arsenic and lead remains to be determined. Further study of such interactions between nutrients, toxicants and bacteria are warranted.

Investigations on the inhibitory effects of cranberries and cranberry-derived constituents on cancer are at an early stage, but are highly encouraging given the emerging positive preclinical effects and limited, but favorable clinical research associated with cranberry administration. Importantly, low concentrations which are achievable when administered in a drink formulation, consumed as food or as a supplement have been reported to elicit cancer inhibitory potential in preclinical models (32-35). Similarly, consumption of cranberry-based products can inhibit Escherichia coli (E. coli) induced urinary tract infections (32, 34, 50, 51).

In contrast, research of black raspberries as cancer inhibitors spans a longer time interval and has progressed to early phase clinical trials in a number of patient cohorts (36-39). Black raspberries can inhibit a broad range of cancers in preclinical models, including in vivo models of oral, esophageal, colon, breast and skin cancer (37). Promising preclinical results led to clinical evaluations in cancer patients or patients at increased risk for cancer development (36-39). Research investigating black raspberries in the clinical setting report positive effects on preneoplastic lesions or cancers of the head and neck, esophagus and colon (38). Berry treatment can also modulate genes linked to RNA processing and growth factor recycling. For example, in the colon, black raspberries inhibited familial adenomatous polyposis (FAP)-associated polyp progression, demethylated tumor suppressor genes and improved plasma cytokine profiles (39). In Barrett's esophagus patients, black raspberry consumption increased tissue levels of glutathione S-transferase Pi (GST-pi) and decreased 8-isoprostane, a marker of lipid peroxidation and global oxidative stress (36). The precise dose, duration and optimum mode of black raspberry delivery for cancer inhibition remains to be fully elucidated. Still, common themes across studies support that black raspberries can inhibit aberrant proliferation and inflammation, reduce oxidative stress and restore tumor suppressive activity.

\section{Chemopreventive effects of docosahexaenoic acid on inflammation-associated carcinogenesis}

In addition to plant-derived nutrients and bioactive compounds, fish-derived long-chain omega-3 fatty acids have significant implications in the protection against inflammatory diseases, including those linked to pollution and cancer (52). Resolvins are thought to be the active metabolites of omega-3 polyunsaturated fatty acids (PUFAs), and are responsible for facilitating the resolving phase of acute and chronic inflammation. Omega-3 PUFAs and resolvins can also modulate cancer progression (52). In addition to suppression of NFKB, activation of AMPK/SIRT1, and modulation of cyclooxygenase (COX) activity by omega-3 PUFAs, up-regulation of anti-inflammatory lipid mediators such as protectins, maresins and resolvins, appear to be the main mechanisms of antineoplastic effects of omega-3 PUFAs (53).

The growth and metastatic potential of cancer cells are influenced by various neighboring cells that comprise the so-called 'tumor microenvironment'. Therefore, the precise understanding of interactions between cancer cells and the surrounding environment is very essential for the discovery of novel anticancer targets and development of efficient therapeutic and preventive strategies. 
Chronic inflammation plays a multifaceted role in carcinogenesis. Mounting evidence from preclinical and clinical studies suggests that persistent inflammation functions as a driving force in the journey to cancer (54). While inflammation promotes development of cancer, components of the tumor microenvironment, such as tumor cells, stromal cells in surrounding tissue and infiltrated inflammatory/ immune cells (e.g. macrophages) generate an intratumoral inflammatory state by aberrant expression or activation of a distinct set of proinflammatory signaling molecules. Many of these inflammatory mediators, especially cytokines, chemokines and prostaglandins, turn on the angiogenic switches mainly controlled by vascular endothelial growth factor and induce tumor cell-stroma communication (54). This will end up with tumor angiogenesis, metastasis and invasion.

Recently, much attention has been focused on dietderived natural products with cancer preventive and therapeutic potential. Over the past few decades, numerous cancer chemopreventive and cytoprotective natural products have been identified that modulate key redoxsensitive transcription factors, such as NF- $\kappa$ B, STAT3 and Nrf2 mediating pro- or anti-inflammatory signaling (55). For example, some spice ingredients, such as curcumin in turmeric [6], gingerol in ginger, capsaicin in hot chili pepper and diallyl trisulfide in garlic can have chemopreventive effects on experimentally induced carcinogenesis (55). In addition to plant-derived nutrients and bioactive compounds, fish-derived long-chain omega-3 fatty acids have significant implications in the protection against inflammatory diseases and cancer (52). Also, docosahexaenoic acid (DHA), a prototypic omega-3 polyunsaturated fatty acid (PUFA) abundant in fish oil, can inhibit colitis (56) and colon carcinogenesis in mice (57) as well as Helicobacter-induced activation of STAT3 in gastric epithelial cells (58).

The implication of inflammatory cell/tissue damage in the pathophysiology of human cancer and some metabolic disorders has been under intense investigation both at the research level and in clinical practice. Resolution of inflammation is an active coordinated process regulated by a distinct set of endogenous lipid mediators, such as resolvins, protectins aresins and lipoxins which are derived from omega-3 PUFAs. Inflammation is often expressed as "fire within". By properly terminating the inflammation in an early stage, these endogeneous fire extinguishers prevent progression to chronic inflammation. In searching for an efficacious way to prevent or treat inflammation-associated disorders, the understanding of pro-resolving signal transduction pathways and their regulators is also essential. Clearance of apoptotic neutrophils by macrophages, termed "efferocytosis", is an essential event in the resolution of inflammation. Recent studies have demonstrated that resolvin D1 derived from DHA stimulates the efferocytosis by facilitating the degradation of $\mathrm{p} 105$, a precursor of p50 subunit of NF-kB and subsequently predominant formation of p50-p50 homodimer (59). This can hamper the formation and subsequent interaction of p50-p65, the functionally active heterodimer of NF- $\mathrm{kB}$, with the $\kappa \mathrm{B}$ binding site present in the promoter regions of many proinflammatory genes. Resolvin D1 also rescues macrophages from oxidative stress-induced apoptosis during efferocytosis through protein kinase A (PKA)-mediated repression of NADPH oxidase (NOX) signaling and upregulation of anti-apoptotic protein expression (60).

Recent studies on the distinct set of metabolites derived from DHA have revealed the endogenous formation of several novel lipid mediators which have proresolving as well as anti-inflammatory effects (59). Considering that acute inflammation, if not resolved properly and in a timely manner, becomes chronic and can cause various human diseases including cancer it is important to evaluate the chemopreventive and anticarcinogenic potential of those proresolving as well as anti-inflammatory substances that originate from DHA (59). With respect to negligible adverse effects of a correct supplementation of DHA, future development of therapeutically relevant, stable synthetic analogs of DHA and its metabolites capable of activating proresolving signal transduction while inhibiting unresolved inflammation merits further investigations (61).

\section{Nutrition or diet-derived bioactive compounds can modulate environ- mental insults}

While a sedentary lifestyle and/or poor dietary habits can exacerbate the harmful effects of exposure to environmental pollutants, much emerging evidence suggests that positive lifestyle changes (e.g. healthful nutrition, exercise or increased physical activity) can modulate or reduce the toxicity of hazardous chemicals $(62,63)$. Diet can either increase or reduce the harmful effects associated with exposure to environmental pollutants. There is a significant need to further explore the paradigm of nutrition in environmental toxicology and to improve our understanding of the relationship between lifestyle modifications and toxicant-induced diseases. 


\section{In vivo evidence of nutritional modulation of environmental insults}

\section{Modulation of PCB toxicity by dietary polyphenols}

The paradigm of nutrition as a key factor modifying environmental chemical (e.g. PCB) toxicity is of considerable interest to populations at risk, i.e. populations residing near hazardous waste sites or areas of contamination and populations with poor dietary habits. This is even more relevant when there also is a high incidence of infectious diseases and malnutrition and it is well known that almost all nutrients in the diet play a crucial role in host resistance to infection (64). Exposure to environmental pollutants during vulnerable periods of life, i.e. during development and early childhood, can trigger more severe onset of non-communicable diseases later in life (25). Most importantly, the added stress of infectious diseases, poverty and malnutrition can compound the vulnerability to environmental chemical-mediated disease pathologies. For example, children around the world, and especially in many developing countries, are at higher risk for the development of diseases due to exposure to toxic substances in the environment (65) and especially persistent organic toxicants or POPs. There is sufficient evidence that POPs contribute to inflammation by activating oxidative stress-sensitive transcription factors such as NF- $\mathrm{\kappa B}(66)$. For example, studies suggest that PCBs, and in particular coplanar PCBs, can increase cellular oxidative stress and induce inflammatory molecules, such as inflammatory cytokines, chemokines and adhesion molecules, in the vascular endothelium, which are metabolic events that foster an inflammatory response and the pathology of atherosclerosis (63). Importantly, exposure to dioxinlike PCBs can elicit epigenetic modifications related to activation of NF- $\mathrm{KB}$ (67). Epigenetic modifications of DNA and histones alter cellular phenotypes without changing genetic codes. A recent study demonstrated that endothelial cell dysfunction induced by exposure to dioxin-like PCBs is mediated in part through histone modifications (68). Increased accumulation of the histone demethylase JMJD2B in the $\mathrm{p} 65$ promoter of NF- $\mathrm{KB}$ led to a depletion of H3K9me3 repression marks (67). This may account for the observed up-regulation of $\mathrm{p} 65$ and associated inflammatory genes. These data suggest that dioxin-like PCBs may exert endothelial cell toxicity through changes in histone modifications $(67,68)$.
An accumulating body of evidence clearly shows that nutrition can modulate PCB toxicity $(62,63)$. For example, specific fatty acids present in many oils, such as linoleic acid (the parent omega- 6 fatty acid), can amplify PCB toxicity in vascular endothelial cells, an event which can be down-regulated by vitamin E (63). Additionally, polyphenols and omega-3 PUFAs have been shown to decrease toxicant-induced maladies including liver diseases, tumor formation and growth and endothelial cell activation (63). Most importantly, plant-derived flavonoids such as epigallocatechin-3-gallate (EGCG), can decrease oxidative stress and inflammation, and this observed protection may be in part controlled via epigenetic mechanisms (68). Understanding of the epigenetic control mechanisms of pollutant-induced diseases will allow for more focused therapeutic and preventative measures which can be implemented during developmental or early phases in life and thus may be highly applicable to the fields of public health and risk assessment.

\section{Clinical evidence of nutritional modulation of environmental insults}

\section{Folic acid supplementation modulates arsenic methylation and blood arsenic levels}

The World Health Organization (WHO) estimates that more than 140 million people in over 70 countries worldwide, including regions within the United States are exposed to arsenic-contaminated drinking water. Ingested inorganic arsenic undergoes methylation to mono- and di-methylated arsenical species, which facilitates renal arsenic elimination. This process relies on folate-dependent one-carbon metabolism for the synthesis of the universal methyl donor, s-adenosylmethionine (SAM). In folate deficient individuals, it was found that methylation of arsenic to the di-methylated species is augmented by folic acid supplementation (400 $\mu \mathrm{g} /$ day, i.e. the US Recommended Dietary Intake) thereby lowering blood arsenic $(69,70)$. In other studies, folate deficiency, assessed by plasma folate concentrations, has been associated with a risk for arsenic-induced skin lesions and urothelial carcinoma $(71,72)$. In addition to folate, choline and betaine can also provide methyl groups for SAM biosynthesis, although these micronutrients have been less well studied with regard to arsenic methylation.

A more recent clinical trial in Bangladesh was conducted to determine whether 400 or $800 \mu \mathrm{g}$ folic acid 
supplementation can increase arsenic methylation and thus lower blood arsenic in a random sample of arsenicexposed Bangladeshi adults (73). Most of the participants were not folate deficient. The major findings of this study included that treatment with $800 \mu \mathrm{g}$ folic acid lowered blood arsenic to a greater extent than placebo (73). Folic acid supplementation rapidly and significantly increased arsenic methylation, which was sustained in those participants who continued their folic acid regimen, but was not sustained in those who switched to placebo for 12 weeks, illustrating the importance of maintaining folate nutritional status. Future studies will be needed to determine if folate supplementation can prevent or potentially reverse clinical signs of arsenic toxicity.

While fortification of food with folic acid is mandated by law in 86 countries and can virtually eradicate folate deficiency, the 10 countries with the most significant problems of arsenic-contaminated drinking water do not have mandatory folate fortification programs (74). Future studies should evaluate folate nutritional status and arsenic methylation capacity in other countries having significant problems of arsenic-contaminated drinking water.

\section{Epidemiological studies of healthful nutrition and protection against environmental insults}

Data from the National Health and Nutrition Examination Survey (NHANES) 2003-2004 predicted a reduced probability of dioxin-like PCB-associated risk for type 2 diabetes in people with higher dietary intake of fruit and vegetables, as reflected by serum carotenoid concentrations (75). In another study among older Chinese adults, it was found that long-term exposure to ambient fine particle pollution contributed significantly to hypertension in this population group (76). It was also observed that higher consumption of fruit might mitigate, whereas overweight and obesity could enhance this pollutioninduced risk of hypertension (76). Most of all, interactions between nutrition and air pollution are particularly critical during developmental phases, and thus could possibly alter the odds of congenital heart defects in offspring (77).

Many POPs are lipophilic and can accumulate within adipose tissues of exposed individuals. Thus, nutritional intervention to promote excretion and reduction of body burden can also be important to lower disease risks. This has been demonstrated in a case study of accidental exposure to Aroclor 1254, where treatment with the dietary fat substitute olestra for over 2 years normalized initial symptoms of type 2 diabetes, mixed hyperlipidemia and chloracne (78). A follow-up study with residents residing near a highly contaminated site with POPs (e.g. PCBs) demonstrated that foods enriched with olestra can safely reduce body burdens of PCBs (79). It has been suggested that olestra, and possibly plant-derived polyphenols, can enhance the excretion rate in individuals exposed to high concentrations of PCBs through interference with the enterohepatic circulation of these pollutants $(79,80)$.

\section{Conclusion}

The paradigm of nutrition as a key factor modifying environmental chemical (e.g. PCB) toxicity is of considerable interest to populations at risk, such as populations residing near hazardous waste sites or areas of contamination and populations with poor dietary habits. This is even more relevant when there also is a high incidence of infectious diseases and malnutrition, as it is well known that almost all nutrients in the diet play a crucial role in host resistance to infection (64). This may be especially relevant in Southeast Asian regions of the world where infectious diseases, poverty and malnutrition are prevalent. Furthermore, the role of nutrition in cumulative risk assessment is being further substantiated not only as an important modulator of inflammatory and antioxidant pathways, especially associated with environmental insults, but also due to its link to the pathologies of non-communicable or chronic diseases (81). Diets rich in antioxidants and antiinflammatory nutrients can improve health and decrease vulnerability to additional chemical stressors (Figure 1). Thus, healthful nutrition intervention may be most effective if it is considered as early in life as possible. This is especially prudent in children who are malnourished and also suffer from infections (64). In fact, a healthy diet could markedly buffer the body against chemical, biological and physical stressors that humans are exposed to on a daily basis. In summary, positive lifestyle changes, including healthful dietary behaviors and food choices, can potentially reduce health risks associated with exposure to hazardous substances or reduce the overall vulnerability to environmental insults. This suggests that nutritional interventions may provide the most sensible prevention strategy against diseases associated with many environmental toxic insults. Looking forward, it will be important to conduct more human clinical studies to document and quantify the potential benefits of nutritional interventions in reducing incidence and progression of disease. 
Acknowledgments: The authors wish to thank Angela Spivey, a contractor for MDB Inc., supporting the NIEHS Superfund Research Program, for valuable editorial assistance.

\section{Author Statement}

Research funding: Research reported in this article was supported in part by NIEHS/NIH grant R13ES027338. The content is solely the responsibility of the authors and does not necessarily represent the official views of NIH. Conflict of interest: Authors state no conflict of interest. Informed consent: Informed consent is not applicable. Ethical approval: The conducted research is not related to either human or animal use.

\section{References}

1. Ngo AD, Taylor R, Roberts CL, Nguyen TV. Association between Agent Orange and birth defects: systematic review and metaanalysis. Int J Epidemiol 2006;35:1220-30.

2. Pavuk M, Michalek JE, Schecter A, Ketchum NS, Akhtar FZ, et al. Did TCDD exposure or service in Southeast Asia increase the risk of cancer in air force Vietnam veterans who did not spray agent orange? J Occup Environ Med 2005;47:335-42.

3. Patterson AT, Kaffenberger BH, Keller RA, Elston DM. Skin diseases associated with Agent Orange and other organochlorine exposures. J Am Acad Dermatol 2016;74:143-70.

4. McIntyre D, Ranson MK, Aulakh BK, Honda A. Promoting universal financial protection: evidence from seven low- and middleincome countries on factors facilitating or hindering progress. Health Res Policy Syst 2013;11:36.

5. Usfar AA, Lebenthal E, Atmarita, Achadi E, Soekirman, et al. Obesity as a poverty-related emerging nutrition problems: the case of Indonesia. Obes Rev 2010;11:924-8.

6. Bates M, Marais BJ, Zumla A. Tuberculosis comorbidity with communicable and noncommunicable diseases. Cold Spring Harb Perspect Med 2015;5:pii: a017889.

7. Hotez PJ, Ehrenberg JP. Escalating the global fight against neglected tropical diseases through interventions in the Asia Pacific region. Adv Parasitol 2010;72:31-53.

8. Safronetz D, Feldmann H, de Wit E. Birth and pathogenesis of rogue respiratory viruses. Annu Rev Pathol 2015;10:449-71.

9. Friel S, Baker PI. Equity, food security and health equity in the Asia Pacific region. Asia Pac J Clin Nutr 2009;18:620-32.

10. Weinberger KM, Easdown WJ, Yang RY, Keatinge JD. Food crisis in the Asia-Pacific region. Asia Pac J Clin Nutr 2009;18:507-15.

11. Dar AA, Arumugam N. Lignans of sesame: purification methods, biological activities and biosynthesis-a review. Bioorg Chem 2013;50:1-10.

12. Kamal-Eldin A, Moazzami A, Washi S. Sesame seed lignans: potent physiological modulators and possible ingredients in functional foods \& nutraceuticals. Recent Pat Food Nutr Agric 2011;3:17-29.

13. Majdalawieh AF, Massri M, Nasrallah GK. A comprehensive review on the anti-cancer properties and mechanisms of action of sesamin, a lignan in sesame seeds (Sesamum indicum). Eur J Pharmacol 2017;815:512-21.

14. Xu P, Cai F, Liu X, Guo L. Sesamin inhibits lipopolysaccharideinduced proliferation and invasion through the p38-MAPK and NF-kappaB signaling pathways in prostate cancer cells. Oncol Rep 2015;33:3117-23.

15. Pianjing P, Thiantanawat A, Rangkadilok N, Watcharasit $P$, Mahidol C, et al. Estrogenic activities of sesame lignans and their metabolites on human breast cancer cells. J Agric Food Chem 2011;59:212-21.

16. Ahn YO. Diet and stomach cancer in Korea. Int J Cancer 1997; Suppl 10:7-9.

17. Hajat A, Allison M, Diez-Roux AV, Jenny NS, Jorgensen NW, et al. Long-term exposure to air pollution and markers of inflammation, coagulation, and endothelial activation: a repeat-measures analysis in the Multi-Ethnic Study of Atherosclerosis (MESA). Epidemiology 2015;26:310-20.

18. Oyinloye BE, Ajiboye BO, Ojo OA, Nwozo So, Kappo AP. Cardioprotective and antioxidant influence of aqueous extracts from sesamum indicum seeds on oxidative stress induced by cadmium in Wistar rats. Pharmacogn Mag 2016;12:S170-4.

19. Wichitsranoi J, Weerapreeyakul N, Boonsiri P, Settasatian C, Settasatian N, et al. Antihypertensive and antioxidant effects of dietary black sesame meal in pre-hypertensive humans. Nutr J 2011;10:82.

20. Alipoor B, Haghighian MK, Sadat BE, Asghari M. Effect of sesame seed on lipid profile and redox status in hyperlipidemic patients. Int J Food Sci Nutr 2012;63:674-8.

21. Frommherz L, Bub A, Hummel E, Rist MJ, Roth A, et al. Agerelated changes of plasma bile acid concentrations in healthy adults-results from the cross-sectional KarMeN study. PLoS One 2016;11:e0153959.

22. Rangkadilok N, Worasuttayangkurn L, Bennett RN, Satayavivad J. Identification and quantification of polyphenolic compounds in Longan (Euphoria longana Lam.) fruit. J Agric Food Chem 2005;53:1387-92.

23. Rangkadilok N, Sitthimonchai S, Worasuttayangkurn L, Mahidol C, Ruchirawat $M$, et al. Evaluation of free radical scavenging and antityrosinase activities of standardized longan fruit extract. Food Chem Toxicol 2007;45:328-36.

24. Kunworarath N, Rangkadilok N, Suriyo T, Thiantanawat A, Satayavivad J. Longan (Dimocarpus longan Lour.) inhibits lipopolysaccharide-stimulated nitric oxide production in macrophages by suppressing NF-kappaB and AP-1 signaling pathways. J Ethnopharmacol 2016;179:156-61.

25. Suk WA, Ahanchian H, Asante KA, Carpenter DO, Diaz-Barriga F, et al. Environmental pollution: an under-recognized threat to children's health, especially in low- and middle-income countries. Environ health perspect 2016;124:A41-5.

26. Gerhauser C, Alt A, Heiss E, Gamal-Eldeen A, Klimo K, et al. Cancer chemopreventive activity of Xanthohumol, a natural product derived from hop. Mol Cancer Ther 2002;1:959-69.

27. Pichler C, Ferk F, Al-Serori H, Huber W, Jager W, et al. Xanthohumol prevents DNA damage by dietary carcinogens: results of a human intervention trial. Cancer Prev Res (Phila) 2017;10:153-60.

28. Moller P, Wallin H, Vogel U, Autrup H, Risom L, et al. Mutagenicity of 2-amino-3-methylimidazo[4,5-f]quinoline in colon and liver of Big Blue rats: role of DNA adducts, strand breaks, DNA repair and oxidative stress. Carcinogenesis 2002;23:1379-85. 
29. Jongthawin J, Techasen A, Loilome W, Yongvanit P, Namwat N. Anti-inflammatory agents suppress the prostaglandin E2 production and migration ability of cholangiocarcinoma cell lines. Asian Pac J Cancer Prev 2012;13 Suppl:47-51.

30. Pinto C, Duque AL, Rodriguez-Galdon B, Cestero JJ, Macias P. Xanthohumol prevents carbon tetrachloride-induced acute liver injury in rats. Food Chem Toxicol 2012;50:3405-12.

31. Ferk F, Misik M, Nersesyan A, Pichler C, Jager W, et al. Impact of xanthohumol (a prenylated flavonoid from hops) on DNA stability and other health-related biochemical parameters: Results of human intervention trials. Mol Nutr Food Res 2016;60:773-86.

32. Blumberg JB, Camesano TA, Cassidy A, Kris-Etherton P, Howell $A$, et al. Cranberries and their bioactive constituents in human health. Adv Nutr 2013;4:618-32.

33. Kresty LA, Weh KM, Zeyzus-Johns B, Perez LN, Howell AB. Cranberry proanthocyanidins inhibit esophageal adenocarcinoma in vitro and in vivo through pleiotropic cell death induction and $\mathrm{PI3K} / \mathrm{AKT} / \mathrm{mTOR}$ inactivation. Oncotarget 2015;6:33438-55.

34. Pappas E, Schaich KM. Phytochemicals of cranberries and cranberry products: characterization, potential health effects, and processing stability. Crit Rev Food Sci Nutr 2009;49:741-81.

35. Weh KM, Clarke J, Kresty LA. Cranberries and cancer: an update of preclinical studies evaluating the cancer inhibitory potential of cranberry and cranberry derived constituents. Antioxidants (Basel) 2016;5:pii: E27.

36. Kresty LA, Fromkes JJ, Frankel WL, Hammond CD, Seeram NP, et al. A phase I pilot study evaluating the beneficial effects of black raspberries in patients with Barrett's esophagus. Oncotarget 2016. Available at: http://www.oncotarget.com/ abstract/10457.

37. Kresty LA, Mallery SR, Stoner GD. Black raspberries in cancer clinical trials: Past, present and future. J Berry Res 2016;6:251-61.

38. Mallery SR, Tong M, Shumway BS, Curran AE, Larsen PE, et al. Topical application of a mucoadhesive freeze-dried black raspberry gel induces clinical and histologic regression and reduces loss of heterozygosity events in premalignant oral intraepithelial lesions: results from a multicentered, placebo-controlled clinical trial. Clin Cancer Res 2014;20:1910-24.

39. Wang LS, Burke CA, Hasson H, Kuo CT, Molmenti CL, et al. A phase Ib study of the effects of black raspberries on rectal polyps in patients with familial adenomatous polyposis. Cancer Prev Res (Phila) 2014;7:666-74.

40. Weh KM, Howell AB, Kresty LA. Expression, modulation, and clinical correlates of the autophagy protein Beclin-1 in esophageal adenocarcinoma. Mol Carcinog 2016;55:1876-85.

41. Khanna S, Gharpure AS. Petroleum carcinogenicity and aerodigestive tract: in context of developing nations. Cureus 2017;9:e1202.

42. Blumberg JB, Basu A, Krueger CG, Lila MA, Neto CC, et al. Impact of cranberries on gut microbiota and cardiometabolic health: proceedings of the cranberry health research conference 2015 . Adv Nutr 2016;7:759S-70S.

43. Anhe FF, Roy D, Pilon G, Dudonne S, Matamoros S, et al. A polyphenol-rich cranberry extract protects from diet-induced obesity, insulin resistance and intestinal inflammation in association with increased Akkermansia spp. population in the gut microbiota of mice. Gut 2015;64:872-83.

44. Safavi M, Sabourian R, Foroumadi A. Treatment of Helicobacter pylori infection: Current and future insights. World J Clin Cases 2016;4:5-19.
45. Vale FF, Oleastro M. Overview of the phytomedicine approaches against Helicobacter pylori. World J Gastroenterol 2014;20:5594-609.

46. Bystrom LM, Guzman ML, Rivella S. Iron and reactive oxygen species: friends or foes of cancer cells? Antioxid Redox Signal 2014;20:1917-24.

47. Guo M, Perez C, Wei Y, Rapoza E, Su G, et al. Iron-binding properties of plant phenolics and cranberry's bio-effects. Dalton Trans 2007:4951-61.

48. Chaston TB, Watts RN, Yuan J, Richardson DR. Potent antitumor activity of novel iron chelators derived from di-2-pyridylketone isonicotinoyl hydrazone involves fenton-derived free radical generation. Clin Cancer Res 2004;10:7365-74.

49. Kalinowski DS, Richardson DR. Future of toxicology-iron chelators and differing modes of action and toxicity: the changing face of iron chelation therapy. Chem Res Toxicol 2007;20:715-20.

50. Ledda A, Belcaro G, Dugall M, Riva A, Togni S, et al. Highly standardized cranberry extract supplementation (Anthocran $(R)$ ) as prophylaxis in young healthy subjects with recurrent urinary tract infections. Eur Rev Med Pharmacol Sci 2017;21:389-93.

51. Luis A, Domingues F, Pereira L. Can cranberries contribute to reduce the incidence of urinary tract infections? A systematic review with meta-analysis and trial sequential analysis of clinical trials. J Urol 2017;198:614-21.

52. Moro K, Nagahashi M, Ramanathan R, Takabe K, Wakai T. Resolvins and omega three polyunsaturated fatty acids: Clinical implications in inflammatory diseases and cancer. World J Clin Cases 2016;4:155-64.

53. Nabavi SF, Bilotto S, Russo GL, Orhan IE, Habtemariam S, et al. Omega-3 polyunsaturated fatty acids and cancer: lessons learned from clinical trials. Cancer Metastasis Rev 2015;34: 359-80.

54. Kundu JK, Surh YJ. Inflammation: gearing the journey to cancer. Mutat Res 2008;659:15-30.

55. Surh YJ. Cancer chemoprevention with dietary phytochemicals. Nat Rev Cancer 2003;3:768-80.

56. Yum HW, Kang JX, Hahm KB, Surh YJ. Constitutive omega-3 fatty acid production in fat-1 transgenic mice and docosahexaenoic acid administration to wild type mice protect against 2,4,6-trinitrobenzene sulfonic acid-induced colitis. Biochem Biophys Res Commun 2017;487:847-55.

57. Takahashi M, Fukutake M, Isoi T, Fukuda K, Sato H, et al. Suppression of azoxymethane-induced rat colon carcinoma development by a fish oil component, docosahexaenoic acid (DHA). Carcinogenesis 1997;18:1337-42.

58. Ji HG, Piao JY, Kim SJ, Kim DH, Lee HN, et al. Docosahexaenoic acid inhibits Helicobacter pylori-induced STAT3 phosphorylation through activation of PPARgamma. Mol Nutr Food Res 2016;60:1448-57.

59. Lee HN, Kundu JK, Cha YN, Surh YJ. Resolvin D1 stimulates efferocytosis through p50/p50-mediated suppression of tumor necrosis factor-alpha expression. J Cell Sci 2013;126:4037-47.

60. Lee HN, Surh YJ. Resolvin D1-mediated NOX2 inactivation rescues macrophages undertaking efferocytosis from oxidative stress-induced apoptosis. Biochem Pharmacol 2013;86:759-69.

61. Yum HW, Na HK, Surh YJ. Anti-inflammatory effects of docosahexaenoic acid: Implications for its cancer chemopreventive potential. Semin Cancer Biol 2016;40-41:141-59.

62. Petriello MC, Newsome BJ, Dziubla TD, Hilt JZ, Bhattacharyya D, et al. Modulation of persistent organic pollutant toxicity through 
nutritional intervention: emerging opportunities in biomedicine and environmental remediation. Sci Total Environ 2014; 491-492:11-6.

63. Petriello MC, Newsome B, Hennig B. Influence of nutrition in PCB-induced vascular inflammation. Environ Sci Pollut Res Int 2014;21:6410-8.

64. Field CJ, Johnson IR, Schley PD. Nutrients and their role in host resistance to infection. J Leukoc Biol 2002;71:16-32.

65. Ruchirawat M, Navasumrit P, Settachan D, Autrup H. Environmental impacts on children's health in Southeast Asia: genotoxic compounds in urban air. Ann N Y Acad Sci 2006;1076:678-90.

66. Hennig B, Meerarani P, Slim R, Toborek M, Daugherty A, et al. Proinflammatory properties of coplanar PCBs: in vitro and in vivo evidence. Toxicol Appl Pharmacol 2002;181:174-83.

67. Liu D, Perkins JT, Petriello MC, Hennig B. Exposure to coplanar $\mathrm{PCBs}$ induces endothelial cell inflammation through epigenetic regulation of NF-kappaB subunit p65. Toxicol Appl Pharmacol 2015;289:457-65.

68. Liu D, Perkins JT, Hennig B. EGCG prevents PCB-126-induced endothelial cell inflammation via epigenetic modifications of NFkappaB target genes in human endothelial cells. J Nutr Biochem 2016;28:164-70.

69. Gamble MV, Liu X, Ahsan H, Pilsner JR, Ilievski V, et al. Folate and arsenic metabolism: a double-blind, placebo-controlled folic acid-supplementation trial in Bangladesh. Am J Clin Nutr 2006;84:1093-101.

70. Gamble MV, Liu X, Slavkovich V, Pilsner JR, Ilievski V, et al. Folic acid supplementation lowers blood arsenic. Am J Clin Nutr 2007;86:1202-9.

71. Huang YK, Pu YS, Chung CJ, Shiue HS, Yang MH, et al. Plasma folate level, urinary arsenic methylation profiles, and urothelial carcinoma susceptibility. Food Chem Toxicol 2008;46:929-38.

72. Pilsner JR, Liu X, Ahsan H, Ilievski V, Slavkovich V, et al. Folate deficiency, hyperhomocysteinemia, low urinary creatinine, and hypomethylation of leukocyte DNA are risk factors for arsenicinduced skin lesions. Environ Health Perspect 2009;117:254-60.

73. Peters BA, Hall MN, Liu X, Parvez F, Sanchez TR, et al. Folic Acid and creatine as therapeutic approaches to lower blood arsenic: a randomized controlled trial. Environ Health Perspect 2015;123:1294-301.

74. Searby L. Folic acid fortification: The current global state of play. Special edition: gender-specific nutrition. nutraingredients. com: William Reed Business Media SAS; 2016.

75. Hofe CR, Feng L, Zephyr D, Stromberg AJ, Hennig B, et al. Fruit and vegetable intake, as reflected by serum carotenoid concentrations, predicts reduced probability of polychlorinated biphenyl-associated risk for type 2 diabetes: National Health and Nutrition Examination Survey 2003-2004. Nutr Res 2014;34:285-93.

76. Lin H, Guo Y, Zheng Y, Di Q, Liu T, et al. Long-term effects of ambient PM2.5 on hypertension and blood pressure and attributable risk among older chinese adults. Hypertension 2017;69:806-12.

77. Stingone JA, Luben TJ, Carmichael SL, Aylsworth AS, Botto LD, et al. Maternal exposure to nitrogen dioxide, intake of methyl nutrients, and congenital heart defects in offspring. Am J Epidemiol 2017;186:719-29.

78. Redgrave TG, Wallace P, Jandacek RJ, Tso P. Treatment with a dietary fat substitute decreased Arochlor 1254 contamination in an obese diabetic male. J Nutr Biochem 2005;16:383-4.

79. Jandacek RJ, Heubi JE, Buckley DD, Khoury JC, Turner WE, et al. Reduction of the body burden of PCBs and DDE by dietary intervention in a randomized trial. J Nutr Biochem 2014;25:483-8.

80. Jandacek RJ. Intervention to reduce PCBs: learnings from a controlled study of Anniston residents. Environ Sci Pollut Res Int 2016;23:2022-6.

81. Hennig B, Ormsbee L, McClain CJ, Watkins BA, Blumberg B, et al. Nutrition can modulate the toxicity of environmental pollutants: implications in risk assessment and human health. Environ Health Perspect 2012;120:771-4. 